\title{
Hybrid Contents Recommendation Service Using LBS and NFC Tagging
}

\author{
Yoondeuk Seo and Jinho Ahn ${ }^{1}$ \\ Dept. of Comp. Scie., Kyonggi Univ., Iuidong, Yeongtong, Suwon 443-760 Gyeonggi, \\ Republic of Korea \\ \{seoyd,jhahn\}@kgu.ac.kr
}

\begin{abstract}
Near Field Communication (NFC) as a promising short range wireless communication technology facilitates mobile phone usage of billions of people throughout the world that offers diverse services ranging from payment and loyalty applications to access keys for offices and houses. And smartphone positioning is an enabling technology used to create new business in the navigation and mobile location-based services (LBS) industries. In this paper, we propose a hybrid contents recommendation service using LBS and NFC technology. The proposed service recommends contents using viewing path information through LBS and viewing exhibits information through NFC. It might recommend the contents which user likes among the tagged information. Furthermore, it can recommend contents related with exhibits which are the user's favorite, but not tagged by the user. So, it recommends appropriately the contents which is right for the user`s taste.
\end{abstract}

Keywords: NFC tagging, LBS, Museum Viewing, Contents Recommendation

\section{Introduction}

NFC as one of the enablers for ubiquitous computing is a "combination of contactless identification and interconnection technologies" [1] which requires bringing two NFC compatible devices close to each other, essentially touching them. In accordance with [2], user first interacts with a smart object (either an NFC tag, NFC reader, or another NFC enabled mobile phone) using her NFC enabled mobile phone (in short: NFC mobile). After touching occurs, NFC mobile may further make use of received data, or may alternatively use provided mobile services such as opening a web page, making a web service connection etc.

Smartphone indoor positioning technology is a boost to the rapidly growing mobile location-based services (LBS) industry. As the latest initiative, the In-Location Alliance, formed by 22 member companies, including Nokia, Qualcomm, Broadcom, etc., [3], was recently launched to drive innovation and market adoption of high-accuracy indoor positioning and related services. The continued development of accurate and reliable LBS will not only improve the experience of smartphone users, but will also create new marketing opportunities. Emerging indoor LBS include social networking, people finders, marketing campaigns, asset tracking, etc. Because most indoor LBS are used by pedestrians, in this work we focus the development of our proposed indoor positioning solution on a pedestrian scenario.

In this paper, we propose a hybrid contents recommendation service using LBS and NFC technology. The proposed service divides an exhibition into the areas through analyzing the

${ }^{1}$ Corresponding author: Tel.:+82-31-249-9674; Fax:+82-31-249-9673. 
Wi-Fi signal strength and collects information that user tagged NFC tag. It recommends contents using viewing path information through LBS and viewing exhibits information through NFC. It might recommend the contents which user likes among the tagged information. Furthermore, it can recommend contents related with exhibits which are the user`s favorite, but not tagged by the user. So, it recommends appropriately the contents which is right for the user`s taste.

\section{Related Work}

NFC technology was jointly developed by Philips and Sony in late 2002 for contactless communications [4]. It is a short-range half duplex communication protocol, which provides easy and secure communication between various devices (Table 1). In accordance with [5], NFC is distinct from far field RF communication that is used in personal area and longerrange wireless networks. NFC relies on inductive coupling between transmitting and receiving devices. The communication occurs between two compatible devices within few centimeters with $13.56 \mathrm{MHz}$ operating frequency [2].

Table 1. Comparison of WPAN Technologies

\begin{tabular}{|c|c|c|c|}
\hline Parameter & Bluetooth & Zigbee & NFC \\
\hline Range & $10-100 \mathrm{~m}$ & $10-100 \mathrm{~m}$ & $4-10 \mathrm{~cm}$ \\
\hline Data Rate & 0.8-2.1 Mbps & $0.02-0.2 \mathrm{Mbps}$ & 0.02-0.4 Mbps \\
\hline Cost & Low & Low & Low \\
\hline Power consumption & High & Medium & Low \\
\hline Spectrum & $2.4 \mathrm{GHz}$ & $2.4 \mathrm{GHz}$ & $13.56 \mathrm{MHz}$ \\
\hline Security & Low & Low & High \\
\hline Network topology & Piconets, scatternets & Star, tree, mesh & One to one \\
\hline Devices per network & 8 & $2-65,000$ & 2 \\
\hline Usability & Moderate, data centric & Easy, data centric & Easy, human centric \\
\hline Personalization & Medium & Low & High \\
\hline Flexibility & High & High & High \\
\hline Setup time & Approx. $6 \mathrm{~s}$ & Approx. $0.5 \mathrm{~s}$ & Less than $0.1 \mathrm{~s}$ \\
\hline
\end{tabular}

The acting two parts of NFC communication is categorized as initiator and target devices [6]. The Initiator is the device that initiates and guides the data exchange process between the parties. The target is the device that responds to the requests made by the initiator. According to Cho et al., [7], NFC protocol distinguishes between two modes of operation, which are active mode and passive mode. In the active communication mode both devices uses their own energy to generate their own RF field to transmit the data. In the passive communication mode only initiator generates the RF field while the target device makes use of the energy that is created by the active device.

There exist three NFC devices, which can involve in NFC communication: NFC mobile, NFC tag, and NFC reader. Table 2 shows the possible interaction styles among those NFC devices. NFC technology operates in three different operating modes: reader/writer, peer-topeer, and card emulation modes where communication occurs between an NFC mobile on one 
side, and an NFC tag, an NFC mobile, and an NFC reader on the other side respectively [8]. Each operating mode uses distinct communication interfaces (i.e., ISO/IEC 14443, FeliCa, NFCIP-1, NFCIP-2 interfaces) on RF layer as well as has different technical, operational and design requirements [9].

Table 2. Interaction Styles of NFC Devices

\begin{tabular}{ll}
\hline Initiator device & Target device \\
\hline NFC mobile & NFC tag \\
NFC mobile & NFC mobile \\
NFC reader & NFC mobile \\
\hline
\end{tabular}

Identity (ID) positioning technology gets user's position through the location of node which is severing the user. The node can be BS, RFID reader, Access Point (AP) and so on. The accuracy of ID positioning depends on the density of positioning nodes. ID positioning technique is often used in BS positioning system and RFID positioning system with low cost and low accuracy.

Geometric positioning technology calculates user's position through measuring the geometry relations between users and positioning nodes. The classic examples of this technique are Time of Arrival (TOA), Time Difference of Arrival (TDOA), Arrival of Angle (AOA) and the integrations of above. Geometric positioning technique is widely applied in positioning systems with BS, UWB, pseudo-lite, lasers and ultrasound. This technology is easy to popularize, but the error will increase while NLOS exists. Taking BS positioning system for example, the positioning error could be up to hundreds of meters for NLOS. Researchers have done a lot of work to mitigate the NLOS error [10,11, 12], and the error can be reduced by $60-90 \%$ in specific environment. But these work still cannot fulfill the demand of meter level accuracy in wide area indoor LBS.

Fingerprint positioning technology based on fingerprint database. The positioning area is divided into grids and the fingerprints in different grids are acquired before positioning. The fingerprints can be TOA, TDOA, RSS, AOA and so on. Fingerprint matching algorithm based on signal measuring results and fingerprint database is applied during positioning. The typical fingerprint matching algorithm consists of k-Nearest Neighbor (KNN) algorithm [13], neural network [14], Support Vector Regression (SVR) [15], Support Vector Machine (SVM) [16], Orthogonal Locality Preserving Projection (OLPP) [17] and so on. Fingerprint positioning technology can mitigate NLOS error effectively. However, this technology is limited by the heavy workload of fingerprint acquisition and the large amount of fingerprint database. This makes fingerprint only be applied to key region and hard to be popularized.

\section{Location Tracking and Tagged Information Extraction}

In this section, we propose a location tracking method and a tagged information extracting method.

First, GPS technology widely used for location tracking has the disadvantage that indoor such as museum is difficult to track because it requires a Line-of-Sight between senders and receivers. Therefore, in this paper, we use the distance measurement method using the Wi-Fi signal strength that is suitable for indoor location tracking $[11,12]$. As shown in Figure 1, the proposed method divides an exhibition into the areas through analyzing the Wi-Fi signal strength. It measures the time that the user views area. By using this measured time, it finds the areas which the user watched. These areas are the user`s viewing path. And it extracts the 
areas which have the viewing time that is more than an average viewing time from all areas. These extracted area are the actual user`s viewing path that is used in this paper.

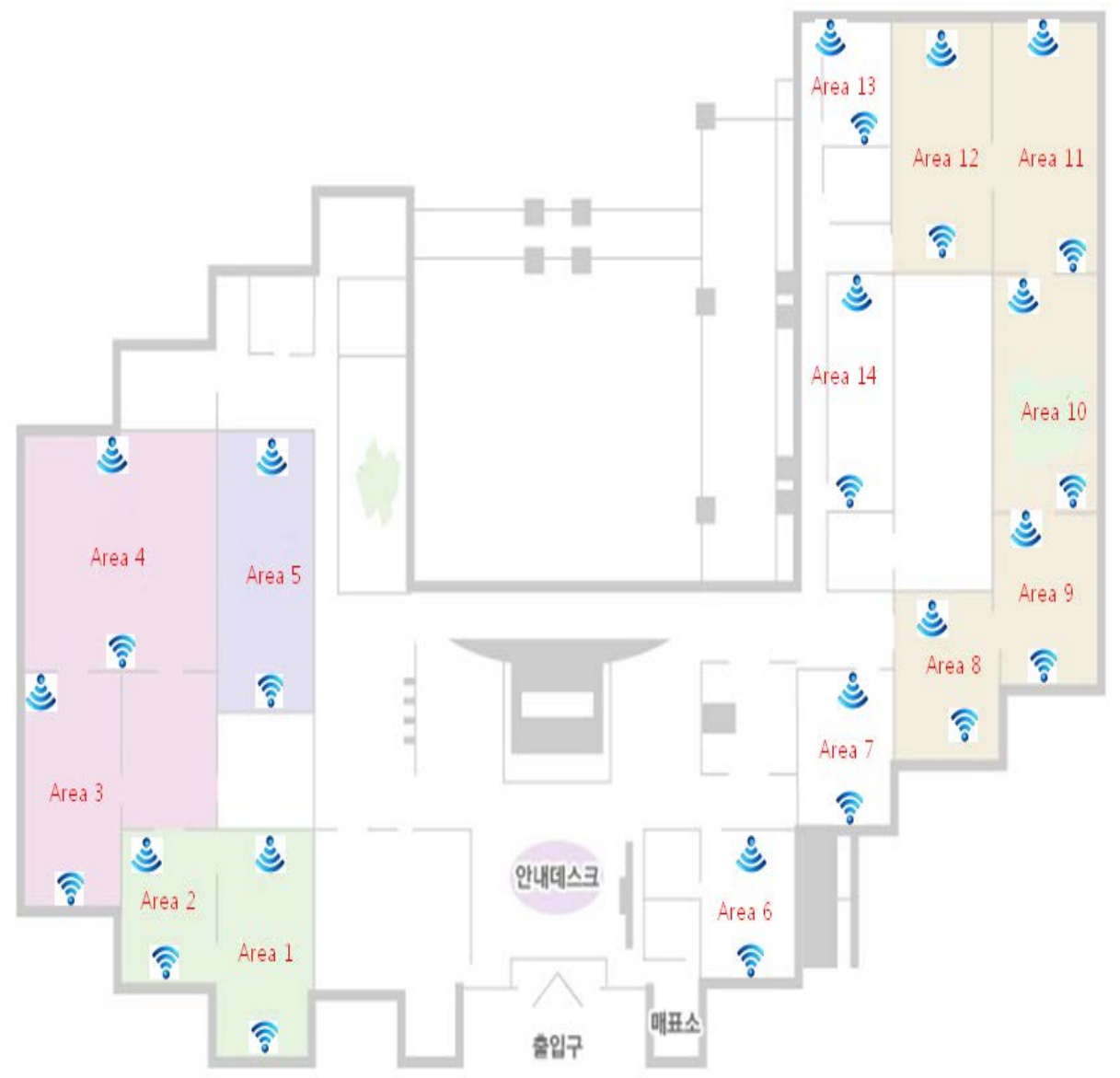

Figure 1. Exhibition Area

Second, we propose a tagged information extracting method using NFC. The NFC tag is adhered to an exhibit in order to provide the exhibit information. The proposed method extracts the exhibits which user tagged.

\section{Contents Recommendation Service based on Viewing Path and Viewing Exhibits}

In this section, we propose the contents recommendation service based on viewing path and viewing exhibits. The proposed service consists of three phase. First, by using the location tracking method, it extracts the user`s viewing path which user watched from all areas. And it measures the viewing path similarity by comparing with other users. Second, by using the tagged information extracting method, it extracts the exhibits which user tagged. And it measures the viewing exhibit similarity by comparing with other users. Finally, it calculates a contents preference using the viewing path similarity and the viewing exhibit similarity.

Figure 2 shows the pseudo-code extracting the viewing path of the user. 


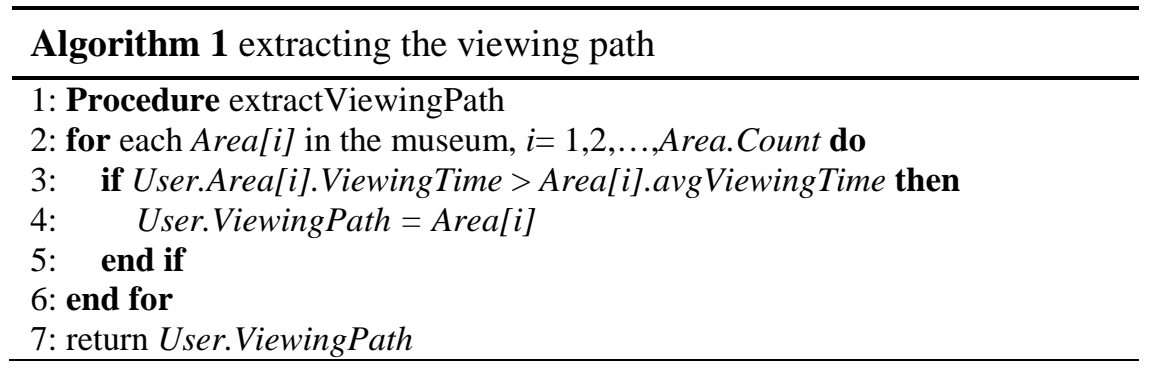

Figure 2. Extracting the Viewing Path of the User

In Figure 2, Area.Count represents the total number of the area and User.Area[i].ViewingTime represents the viewing time when User watched the area $i$. Area[i].avgViewingTime represents the average viewing time of the area $i$. User.ViewingPath represents the viewing path of User.

The similarity between users about watched exhibit can be measured as equation (1).

$$
\operatorname{sim}_{\text {ViewingPath }}(u, p u)=\frac{n\left(V P_{u} \cap V P_{p u}\right)}{n\left(V P_{u}\right)}
$$

In the Equation (1), the argument $u$ presents current user and $p u$ presents previously user who has completed a tour. $V E_{u}$ presents user $u$ of set of watched exhibit and $V E_{p u}$ presents previously user $p u$ of set of watched exhibit. $n\left(V E_{u}\right)$ presents function that find the number of elements of set $V E_{u}$ and $n\left(V E_{u} \cap V E_{p u}\right)$ presents function that find the number of elements of the intersection of $V E_{u}$ and $V E_{p u}$.

Figure 3 shows the pseudo-code extracting the viewing exhibit of the user.

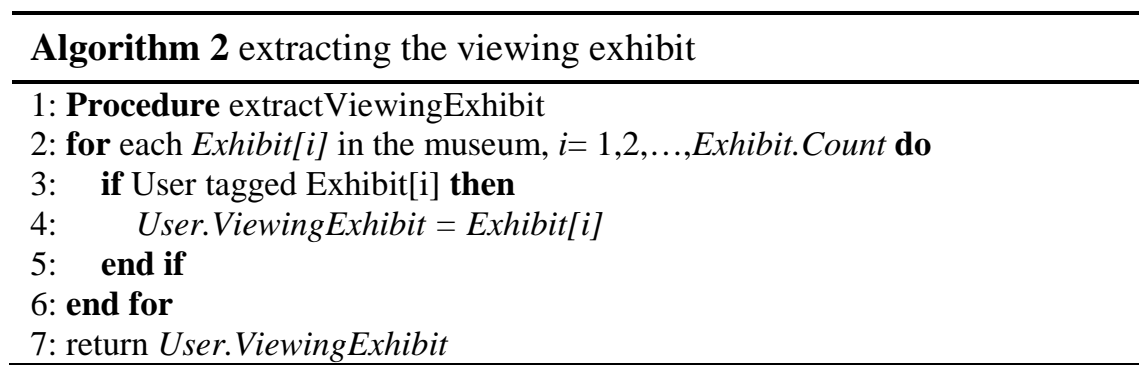

Figure 3. Extracting the Viewing Exhibit of the User

In Figure 3, Exhibit.Count represents the total number of the exhibit.

The similarity between users about watched exhibit can be measured as equation (2).

$$
\operatorname{sim}_{\text {ViewingExhibit }}(u, p u)=\frac{n\left(V E_{u} \cap V E_{p u}\right)}{n\left(V E_{u}\right)}
$$

In the Equation (2), the argument $u$ presents current user and $p u$ presents previously user who has completed a tour. $V E_{u}$ presents the viewing exhibit of user $u$ and $V E_{p u}$ presents the viewing exhibit of previously user $p u$. $n\left(V E_{u}\right)$ presents function that find the number of elements of set $V E_{u}$ and $n\left(V E_{u} \cap N E_{p u}\right)$ presents function that find the number of elements of the intersection of $V E_{u}$ and $V E_{p u}$.

Figure 4 shows the pseudo-code to obtain the contents preference. 


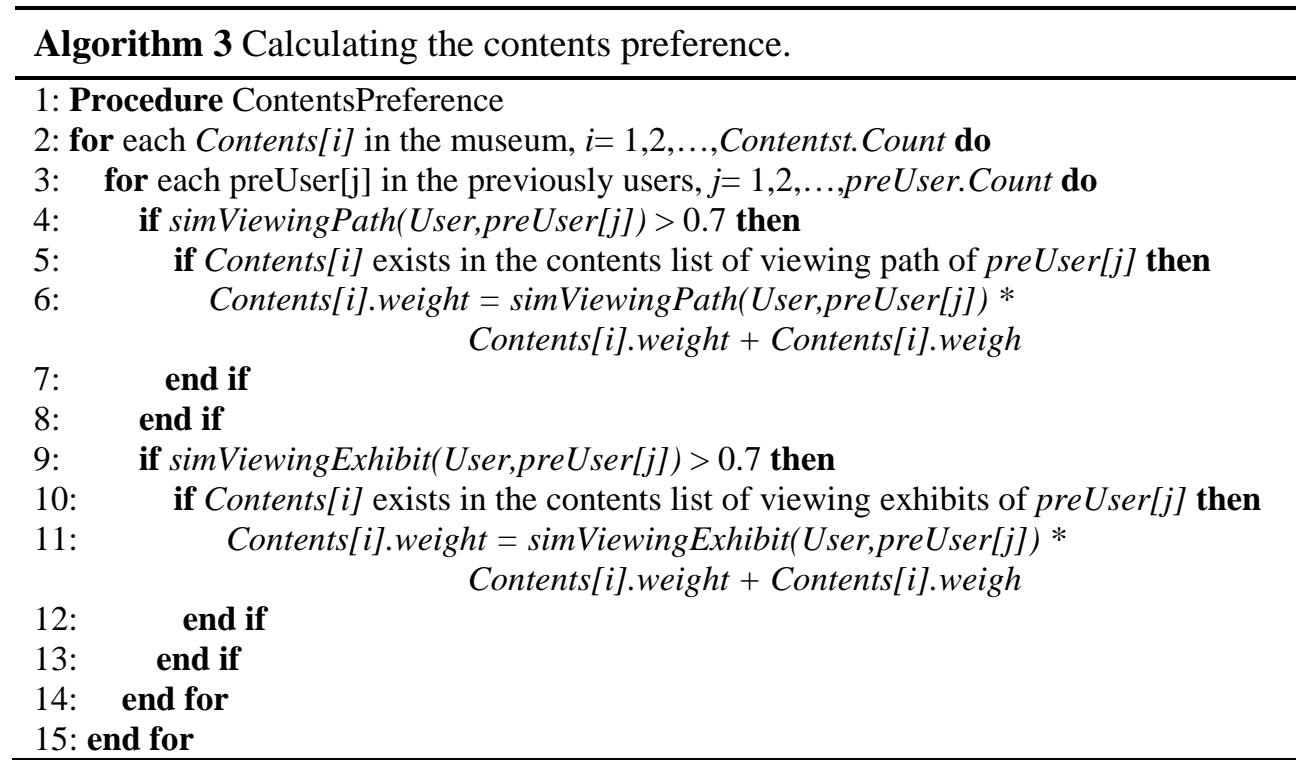

Figure 4. Calculating the Contents Preference

In Figure 4, preUser.Count represents the total number of previously users. preUser[j] represents previously user $j$. simViewingPath(User,preUser[j]) represents the viewing path similarity between User and preUser[j]. If the contents $i$ exists in the contents list of viewing path of preUser[j], contents[i].weight is adjusted by simViewingPath(User,preUser[j]). If the contents $i$ exists in the contents list of viewing exhibits of preUser[j], contents[i].weight is adjusted by simViewingExhibit(User,preUser[j]).

The threshold defines 0.7, meaning very strong relation by Pearson correlation coefficient because the users having the similarity more than the specific value can be seen to have the similar tastes [18].

\section{Performance Evaluation}

In this section, we can show how effectively the proposed service can recommend visitors their favorite contents and solve the problems of existing service. Our experimental environment is in Table 3.

Table 3. Experimental Environment

\begin{tabular}{ll}
\hline Parameter & Value \\
\hline No. of Exhibition Rooms & 10 \\
No. of Area & 30 \\
No. of Exhibits & 60 \\
No. of Contents & 60 \\
No. of Previous Visitors & 100 \\
\hline
\end{tabular}

We assume that the number of exhibition rooms is ten and each room has three areas. So, there are a total of 30 areas. Each area has two exhibits. So, there are a total of 60 exhibits. Each exhibit has one related contents. So, the total number of contents is 60 . The number of previous visitors is 100 . We assume that previous visitors watch at least three exhibitions, stay at least five areas, and watch long at least five exhibits. 
Experiments are performed to find out how the preference values for the present user are changed depending on which services are applied, that is, users similarity method based on tagging patterns [6] and proposed method.

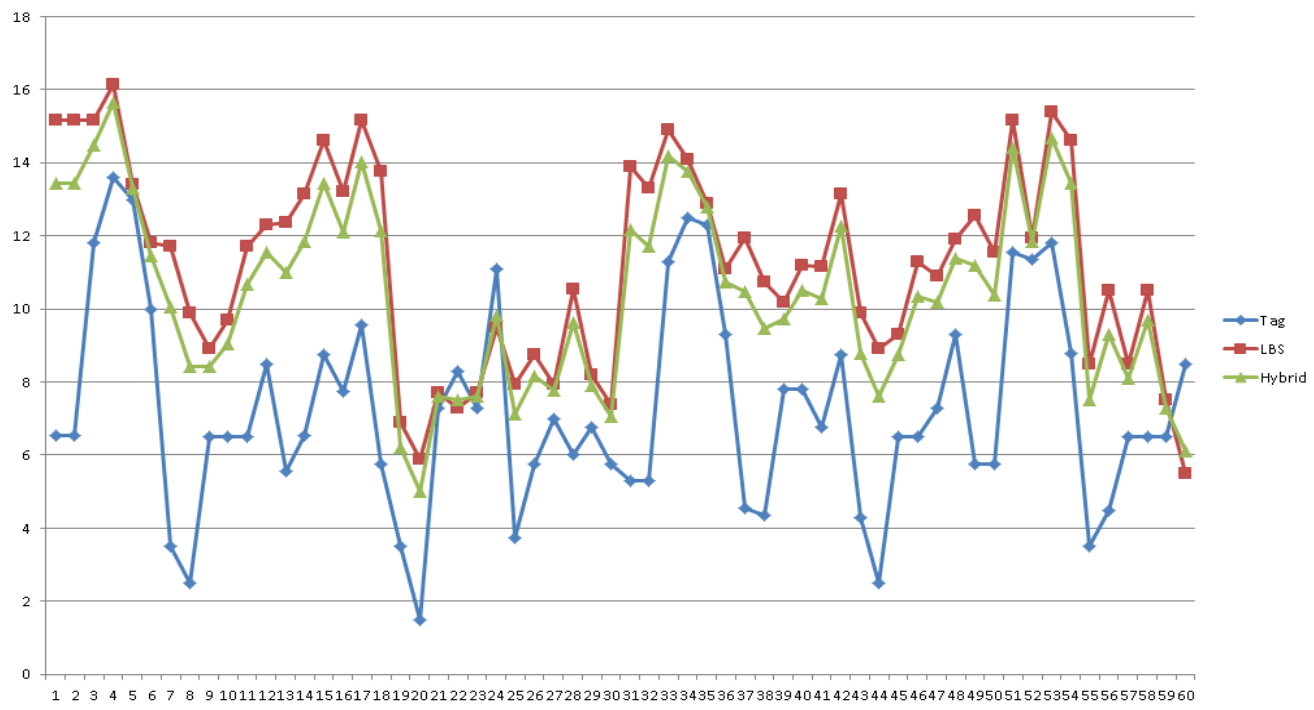

Figure 5. Contents Preference of User A

In order to evaluate the proposed method, we set up three users. The first user has watched rooms 1, 3, 5, 7 and 9 and stayed at area 3, 8, 15, 20 and 27 and tagged exhibits 2, 3, 14, 17 and 27. His or her favorite contents would be 1, 3, 17, 34 and 53. The second user has watched rooms 2, 4, 5, 8 and 10 and stayed at area 5, 12, 14, 24 and 28 and tagged exhibits 4, $10,12,23$ and 28. His or her favorite contents would be 7, 18, 28, 34 and 56 . The last user has watched rooms 1, 2, 4, 8 and 9 and stayed at area 3, 6, 12, 22 and 26 and tagged exhibits 3, 5, 10, 22 and 25. His or her favorite contents would be 4, 5, 24, 44 and 50.

Table 4. Contents Preference Ranking of User A

\begin{tabular}{ccccccc}
\hline \multirow{2}{*}{ Ranking } & \multicolumn{2}{c}{ Tag } & \multicolumn{2}{c}{ LBS } & \multicolumn{2}{c}{ Hybrid(LBS+Tag) } \\
\cline { 2 - 7 } & Contents No. & $\begin{array}{c}\text { Contents } \\
\text { Preference }\end{array}$ & Contents No. & $\begin{array}{c}\text { Contents } \\
\text { Preference }\end{array}$ & Contents No. & $\begin{array}{c}\text { Contents } \\
\text { Preference }\end{array}$ \\
\hline 1 & 4 & 13.6 & 4 & 16.2 & 4 & 14.9 \\
2 & 5 & 13 & 53 & 15.4 & 53 & 13.6 \\
3 & 34 & 12.8 & 1 & 15.2 & 3 & 13.5 \\
4 & 35 & 12.3 & 2 & 15.2 & 34 & 13.4 \\
5 & 53 & 11.8 & 3 & 15.2 & 51 & 13.3 \\
6 & 3 & 11.8 & 17 & 15.2 & 33 & 13.2 \\
7 & 51 & 11.6 & 51 & 15.2 & 5 & 13.2 \\
8 & 33 & 11.6 & 33 & 14.9 & 35 & 12.6 \\
9 & 52 & 11.4 & 15 & 14.6 & 17 & 12.4 \\
10 & 24 & 11.1 & 54 & 14.6 & 54 & 11.7 \\
\hline
\end{tabular}


In the first experiment, Figure 5 shows that each method is applied about the first user. Table 4 shows a contents preference ranking of each method. The contents 17 which is the first user`s favorite contents have low preference values in the tagging pattern based methods, but high preference values in the proposed method. The contents 34 related with exhibit which is tagged by the user have low preference values in the LBS based methods, but high preference values in the proposed method and tagging pattern based methods. In the case of Tag, the user`s favorite contents in the Top 10 is three. In the case of LBS, the user`s favorite contents in the Top 10 is four. In the case of Hybrid(LBS+Tag), the user`s favorite contents in the Top 10 is four.

Table 5. Contents Preference Ranking of User B

\begin{tabular}{ccccccc}
\hline \multirow{2}{*}{ Ranking } & \multicolumn{2}{c}{ Tag } & \multicolumn{2}{c}{ LBS } & \multicolumn{2}{c}{ Hybrid(LBS+Tag) } \\
\cline { 2 - 7 } & Contents No. & $\begin{array}{c}\text { Contents } \\
\text { Preference }\end{array}$ & Contents No. & $\begin{array}{c}\text { Contents } \\
\text { Preference }\end{array}$ & Contents No. & $\begin{array}{c}\text { Contents } \\
\text { Preference }\end{array}$ \\
\hline 1 & 56 & 8.2 & 56 & 11 & 56 & 9.6 \\
2 & 58 & 7.6 & 58 & 10.8 & 58 & 9.2 \\
3 & 16 & 6.5 & 42 & 10.5 & 42 & 8.2 \\
4 & 4 & 6.2 & 4 & 9.5 & 4 & 8 \\
5 & 7 & 6.2 & 16 & 9.5 & 16 & 8 \\
6 & 55 & 6 & 18 & 9.5 & 55 & 7.6 \\
7 & 57 & 5.9 & 55 & 9 & 7 & 7.5 \\
8 & 34 & 5.9 & 7 & 8.8 & 57 & 7.4 \\
9 & 42 & 5.7 & 11 & 8.8 & 18 & 7.2 \\
10 & 1 & 5.7 & 57 & 8.8 & 34 & 7.2 \\
\hline
\end{tabular}

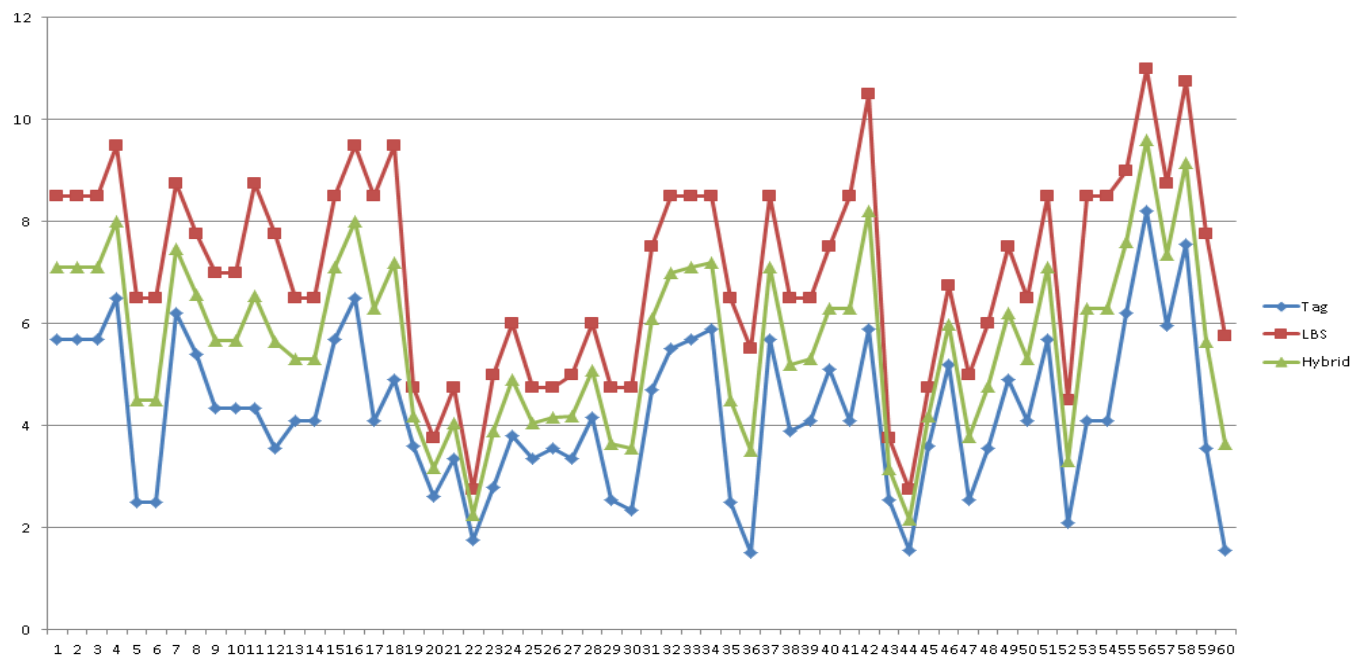

Figure 6. Contents Preference of User B 
In the second experiment, Figure 6 shows that each method is applied about second user. In this case, the three methods almost seem to follow a similar pattern. The contents 18 which is the second user`s favorite contents have low preference values in the tagging pattern based methods, but high preference values in the proposed method. The contents 34 related with exhibit which is tagged by the user have low preference values in the LBS based methods, but high preference values in the proposed method and tagging pattern based methods. In the case of Tag, the user`s favorite contents in the Top 10 is two. In the case of LBS, the user`s favorite contents in the Top 10 is three. In the case of Hybrid, the user`s favorite contents in the Top 10 is four.

Table 6. Contents Preference Ranking of User C

\begin{tabular}{ccccccc}
\hline \multirow{2}{*}{ Ranking } & \multicolumn{2}{c}{ Tag } & \multicolumn{2}{c}{ LBS } & \multicolumn{2}{c}{ Hybrid(LBS+Tag) } \\
\cline { 2 - 7 } & Contents No. & $\begin{array}{c}\text { Contents } \\
\text { Preference }\end{array}$ & Contents No. & $\begin{array}{c}\text { Contents } \\
\text { Preference }\end{array}$ & Contents No. & $\begin{array}{c}\text { Contents } \\
\text { Preference }\end{array}$ \\
\hline 1 & 14 & 8.7 & 2 & 18.4 & 2 & 12.9 \\
2 & 5 & 8.6 & 4 & 18.2 & 17 & 12.6 \\
3 & 49 & 7.6 & 17 & 18.1 & 14 & 12.5 \\
4 & 50 & 7.6 & 3 & 17.6 & 5 & 12.1 \\
5 & 1 & 7.4 & 51 & 17 & 1 & 12.1 \\
6 & 2 & 7.4 & 1 & 16.8 & 53 & 12.1 \\
7 & 53 & 7.4 & 53 & 16.8 & 54 & 12 \\
8 & 54 & 7.4 & 54 & 16.6 & 50 & 11.3 \\
9 & 13 & 7.1 & 14 & 16.3 & 4 & 11.3 \\
10 & 17 & 7.1 & 5 & 15.6 & 3 & 11.3 \\
\hline
\end{tabular}

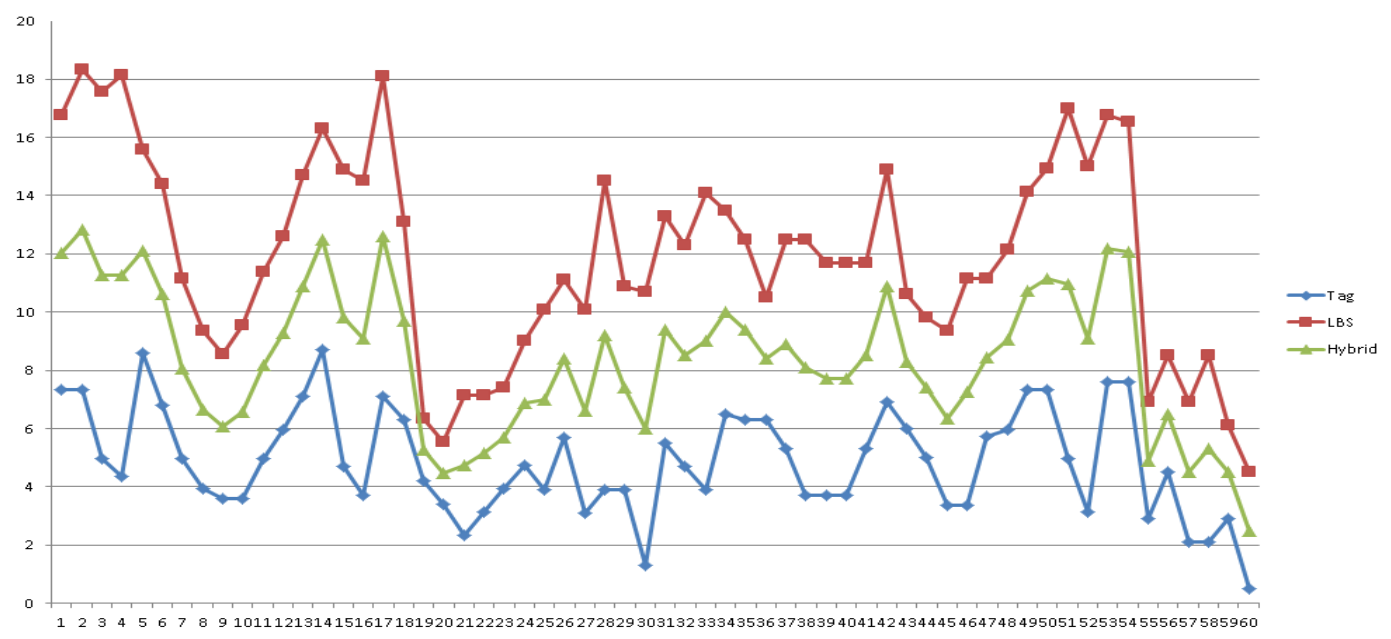

Figure 7. Contents Preference of User C 
In the third experiment, Figure 7 shows that each method is applied about the third user. The contents 4 which is the third user`s favorite contents have low preference values in the tagging pattern based methods, but high preference values in the proposed method. The contents 50 related with exhibit which is tagged by the user have low preference values in the LBS based methods, but high preference values in the proposed method and tagging pattern based methods. In the case of Tag, the user`s favorite contents in the Top 10 is two. In the case of LBS, the user`s favorite contents in the Top 10 is three. In the case of Hybrid, the user`s favorite contents in the Top 10 is three.

Through experiments, we shows that the proposed service recommends the contents which user likes among the tagged information and recommends contents related with exhibits which are the user`s favorite, but not tagged by the user. So, the proposed service recommends appropriately more than tagging pattern and LBS based methods.

\section{Conclusions}

In this paper, we propose a hybrid contents recommendation service using LBS and NFC technology. The proposed service divides an exhibition into the areas through analyzing the Wi-Fi signal strength and collects information that user tagged NFC tag. It recommends contents using viewing path information through LBS and viewing exhibits information through NFC. It might recommend the contents which user likes among the tagged information. Furthermore, it can recommend contents related with exhibits which are the user`s favorite, but not tagged by the user. So, it recommends appropriately the contents which is right for the user`s taste. Through experiments, we show that the proposed service recommends appropriately more than tagging pattern based methods.

\section{References}

[1] D. López-de-Ipiña, J. I. Vazquez and I. Jamardo, "Touch computing: Simplifying human to environment interaction through NFC technology”, 1as Jornadas Científicas sobre RFID, (2007) November 21-23.

[2] V. Coskun, K. Ok and B. Ozdenizci, "Near Field Communication (NFC): From Theory to Practice”, London (2012).

[3] Accurate Mobile Indoor Positioning Industry Alliance. Available online: http://press.nokia.com/2012/08/23/accurate-mobile-indoor-positioning-industry-alliance-called-in-locationto-promotedeployment-of-location-based-indoor-services-and-solutions/ (2012) August 31.

[4] NFC Forum. Available at: http://www.nfc-forum.org.

[5] Y.-S. Lin, D. Sylvester and D. Blaauw, "Near-field communication using phase-locking and pulse signaling for millimeter-scale systems", Proceedings of IEEE 2009 custom integrated circuits conference (CICC), San Jose, CA, USA, (2009) September 13-16.

[6] G. Chavira, S.W. Nava, R. Hervás, J. Bravo and C. Sanchez, “Towards Touching Interaction: A Simple Explicit Input”, Proceedings of the Fourth Annual International Conference on Mobile and Ubiquitous Systems, Philadelphia, PA, USA, (2007) August 6-10.

[7] J. H. Cho, J. Kim, J. W. Kim, K. I Lee, K. D. Ahn and S. H. Kim, “An NFC transceiver with RF-powered RFID transponder mode”, Proceedings of international solid state circuits conference, Jeju, Korea, (2007) November 12-14.

[8] G. Madlmayr, J. Langer and J. Scharinger, "Managing an NFC ecosystem”, Proceedings of 7th international conference on mobile business, Barcelona, Spain, (2008) July 7-8.

[9] ECMA International. ECMA 340: Near field communication interface and protocol (NFCIP-1), Available at: http://www.ecma-international.org/memento/TC47-M.htm, (2004).

[10] M. B. Kjargaard, “A taxonomy for radio location fingerprinting”, Lect. Note. Comput. Sci., vol. 4718, (2007), pp. 139-156.

[11] A. Kushki, K. Plataniotis and A. Venetsanopoulos, "Kernel-based positioning in wireless local area networks”, IEEE Trans. Mobile Comput., vol. 6, (2007), pp. 689-705.

[12] Y. Jie, Y. Qiang and N. Lionel, "Learning adaptive temporal radio maps for signal-strength-based location estimation”, IEEE Trans. Mobile Comput., vol. 7, (2008), pp. 869-886. 
[13] A. Kushki, K. N. Plataniotis and A. Venetsanopoulos, "Intelligent dynamic radio tracking in indoor wireless local area networks”, IEEE Trans. Mobile Comput., vol. 9, (2010), pp. 405419.

[14] A. Au, C. Feng, S. Valaee, S. Reyes, S. Sorour, S. N. Markowitz, D. Gold, K. Gordon and M. Eizenman, "Indoor tracking and navigation using received signal strength and compressive sensing on a mobile device", IEEE Trans. Mobile Comput., in press, (2012).

[15] F. Evennou, F. Marx and E. Novakov, "Map-Aided Indoor Mobile Positioning System Using Particle Filter", Proceeding of 2005 IEEE Wireless Communications and Networking Conference, New Orleans, LA, USA, (2005) March 13-17.

[16] H. Wang, A. Szabo, J. Bamberger, D. Brunn and U. Hanebeck, "Performance Comparison of Nonlinear Filters for Indoor WLAN Positioning”, Proceedings of the International Conference on Information Fusion, Cologne, Germany, (2008) June 30-July 3.

[17] A. Paul and E. Wan, "Wi-Fi Based Indoor Localization and Tracking Using Sigma-point Kalman Filtering Methods", Proceedings of the IEEE/ION Position, Location and Navigation Symposium, Monterey, CA, USA, (2008) May 5-8.

[18] P. Ahlgren, B. Jarneving and R. Rousseau, "Requirements for a cocitation similarity measure with special reference to Pearson's correlation coefficient”, Journal of the American Society for Information Science and Technology, vol. 54, no. 6, (2003).
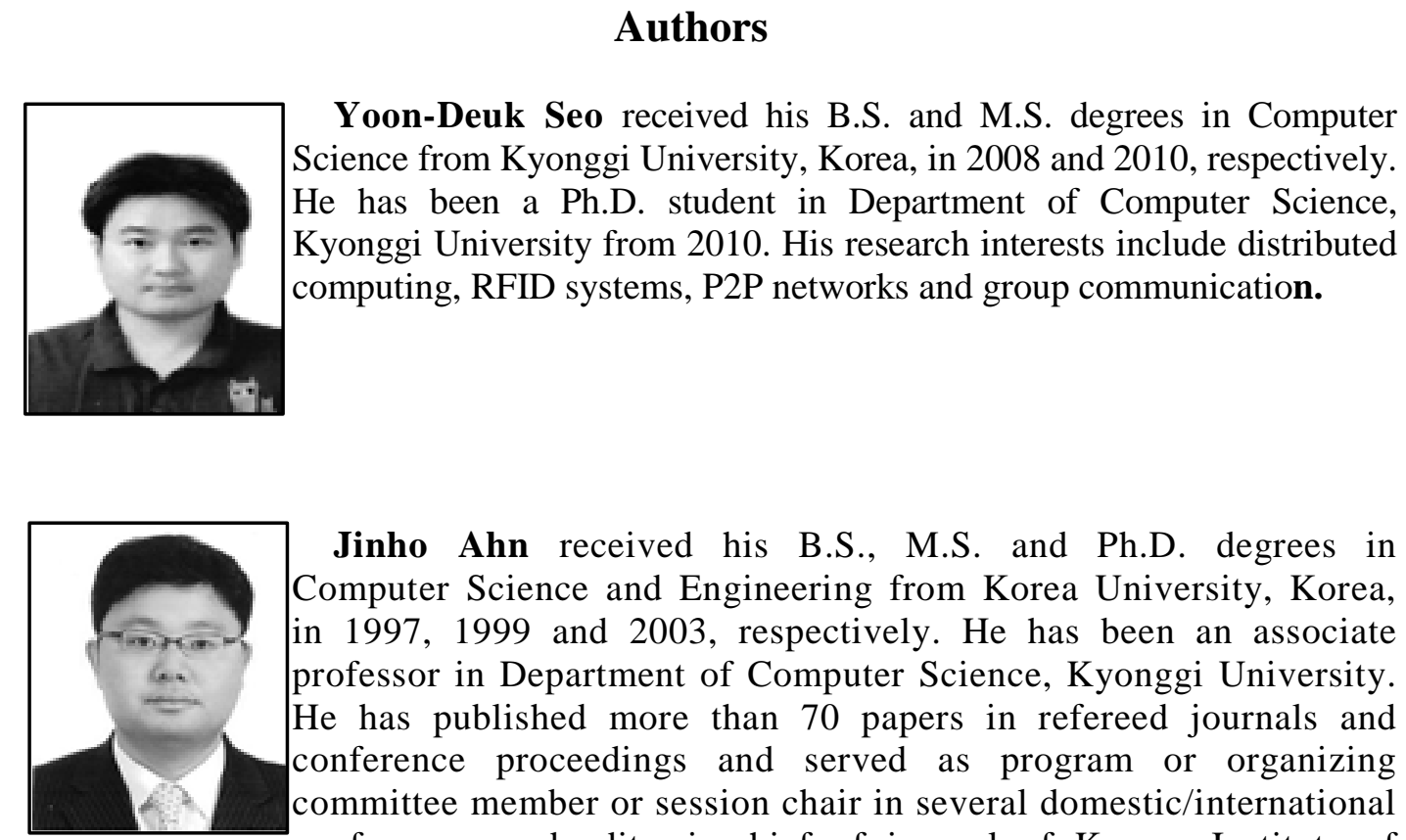

Jinho Ahn received his B.S., M.S. and Ph.D. degrees in Computer Science and Engineering from Korea University, Korea, in 1997, 1999 and 2003, respectively. He has been an associate professor in Department of Computer Science, Kyonggi University. He has published more than 70 papers in refereed journals and conference proceedings and served as program or organizing committee member or session chair in several domestic/international conferences and editor-in-chief of journal of Korean Institute of Information Technology and editorial board member of journal of Korean Society for Internet Information. His research interests include distributed computing, fault-tolerance, sensor networks and mobile agent systems. 
International Journal of Smart Home Vol.7, No.5 (2013) 